\title{
Calidad del agua de una clínica odontológica universitaria de Bogotá
}

\author{
Water quality of a university dental clinic in Bogotá \\ Estupiñán-Torres Sandra Mónica ${ }^{1}$, Ávila de Navia Sara Lilia ${ }^{1}$, Chaves Cárdenas Ana Lucia², \\ Corredor Narváez Ana María ${ }^{2}$
}

\section{Resumen}

Objetivo. Determinar la calidad microbiológica del agua usada en las unidades odontológicas de una clínica universitaria en Bogotá, mediante los indicadores microbiológicos coliformes totales, Escherichia coli, Enterococcus y Pseudomonas. Métodos. Se tomaron muestras de agua de la pieza de mano y de la jeringa triple en 9 unidades odontológicas y tres muestras de los tanques de suministro de agua para un total de 21 muestras. El recuento de microorganismos se realizó mediante la técnica de filtración por membrana. Se aislaron algunos microorganismos presentes en las diferentes muestras a través del kit de pruebas rápidas BBL CRYSTAL. Resultados. El agua no cumple con la Resolución 2115 de 2007 que reglamenta las normas del agua apta para consumo humano. Se aislaron bacterias importantes como Shigella sp. (microorganismo productor de la disentería e indicador de contaminación fecal), Klebsiella y Pseudomonas. Se sugiere implementar medidas correctivas y/o preventivas para minimizar la contaminación y mejorar la calidad del agua usada en estas unidades odontológicas.

Palabras claves: calidad bacteriológica, coliformes totales, Escherichia coli, unidad odontológica.

\section{Abstract}

Objetives. To determine the microbiological quality of the water used in dental units of a University Clinic in Bogotá, through the microbiological indicators like total coliforms, Escherichia coli, Enterococcus and Pseudomonas. Methods. Water samples from hand piece and the triple syringe 9 dental units and three samples of tanks for water supply for a total of 21 samples were taken. The microorganism counting was performed by membrane filtration method according to the Standard Methods. Some microorganisms present in different samples were isolated through the BBL CRYSTAL rapid test kit. Results. The water does not comply with the Resolution 2115 2007, which regulates the water suitable for human consumption standards. Important bacteria such as Shigella sp. (producer of dysentery and a fecal pollution indicator organism), Klebsiella and Pseudomonas were isolated. It is suggested to implement corrective or preventive measures to minimize the pollution and improve the quality of the water used in these dental units.

Keywords: bacteriological quality, coliforms, Escherichia coli, dental unit.

1. Grupo Calidad de aguas. Programa de Bacteriología y Laboratorio Clínico. Universidad Colegio Mayor de Cundinamarca. 


\section{Introducción}

El agua es de fundamental importancia en odontología ya que se usa sobre los pacientes en procedimientos simples como profilaxis o salud oral y procedimientos complejos como cirugías (1). El agua contaminada puede ser fuente de infecciones graves tanto para los pacientes como para el personal de salud (2). La contaminación del agua de las unidades odontológicas puede deberse a diferentes factores como las fuentes de suministro (3) y las biopelículas. Estas se forman por el diámetro reducido de los conductos por los que circula el agua, la baja presión y el estancamiento del agua durante los periodos de inactividad (4). También se puede encontrar contaminación a causa de los microorganismos presentes en la cavidad oral de los pacientes (3). Por lo anterior es muy importante mantener medidas de higiene y aseo en el manejo no solo del material sino de la unidad odontológica e implementar medidas encaminadas a mejorar la calidad del agua utilizada para los procedimientos.

\section{Materiales y métodos}

Se realizó la toma de muestras en la pieza de mano y jeringa triple en 9 unidades odontológicas, dos de estas unidades son utilizadas en procedimientos quirúrgicos. Además, se tomaron muestras de los tanques de suministro y subterráneo. Para hacer los recuentos se utilizó la técnica de filtración por membrana, metodología recomendada por la EPA (Environmental Protection Agency) (5). La identificación de los microorganismos aislados se realizó por medio de pruebas bioquímicas rápidas Crystal BBL.

\section{Resultados}

En la siguiente tabla se encuentran los recuentos obtenidos para la jeringa triple y la pieza de mano de las unidades odontológicas muestreadas.

Tabla 1. Recuento en UFC/100mL de coliformes totales, E.coli, Enterococcus y Pseudomonas en las unidades odontológicas muestreadas.

\begin{tabular}{|c|c|c|c|c|c|}
\hline $\begin{array}{c}\text { Unidad } \\
\text { odontológica }\end{array}$ & Instrumento & $\begin{array}{c}\text { Coliformes } \\
\text { totales }\end{array}$ & E. coli & Enterococcus & Pseudomonas \\
\hline \multirow{2}{*}{1} & JT & 60 & 0 & 0 & 168 \\
\hline & PM & 32 & 0 & 0 & 300 \\
\hline \multirow{2}{*}{5} & $\mathrm{JT}$ & 4 & 0 & 0 & 178 \\
\hline & PM & 170 & 0 & 6 & 290 \\
\hline \multirow{2}{*}{6} & $\mathrm{JT}$ & 40 & 0 & 0 & 300 \\
\hline & PM & 2 & 0 & 2 & 300 \\
\hline \multirow{2}{*}{7} & JT & 40 & 0 & 1 & 300 \\
\hline & PM & 78 & 0 & 27 & 115 \\
\hline \multirow{2}{*}{8} & $\mathrm{JT}$ & 10 & 0 & 1 & 110 \\
\hline & PM & 28 & 0 & 22 & 75 \\
\hline \multirow{2}{*}{13} & $\mathrm{JT}$ & 34 & 0 & 0 & 256 \\
\hline & PM & 40 & 0 & 15 & 63 \\
\hline \multirow{2}{*}{21} & $\mathrm{JT}$ & 12 & 0 & 0 & 230 \\
\hline & PM & 12 & 0 & 1 & 80 \\
\hline \multirow{2}{*}{ SC1 } & $\mathrm{JT}$ & 81 & 0 & 0 & 300 \\
\hline & PM & 4 & 0 & 2 & 300 \\
\hline
\end{tabular}




\begin{tabular}{|l|l|l|l|l|l|}
\hline $\begin{array}{c}\text { Unidad } \\
\text { odontológica }\end{array}$ & \multicolumn{1}{|c|}{ Instrumento } & $\begin{array}{c}\text { Coliformes } \\
\text { totales }\end{array}$ & E. coli & Enterococcus & \multicolumn{1}{|c|}{ Pseudomonas } \\
\hline \multirow{2}{*}{ SC2 } & 62 & 0 & 0 & 67 \\
\cline { 2 - 6 } & JT & 80 & 0 & 2 & 300 \\
\hline JT: jeringa triple, PM: pieza de mano, SC: sala de cirugía \\
\hline
\end{tabular}

Fuente. Elaboración propia.

Se puede observar que los recuentos más altos en los dos instrumentos son de Pseudomonas, seguido por coliformes totales y Enterococcus, no se encontraron recuentos de $E$. coli en ninguna de las muestras procesadas.
En los recuentos de los tanques de abastecimiento se observa que, al igual que en las muestras de las unidades odontológicas, el microorganismo que mayor recuento obtuvo fue Pseudomonas y no se aisló E. coli (Ver tabla 2).

Tabla 2. Recuento en UFC/100mL de coliformes totales, E.coli, Enterococcus y Pseudomonas en los tanques de abastecimiento.

\begin{tabular}{|c|l|l|l|l|}
\hline TANQUE & \multicolumn{1}{|c|}{$\begin{array}{c}\text { Coliformes } \\
\text { totales }\end{array}$} & \multicolumn{1}{|c|}{ E. coli } & Enterococcus & \multicolumn{1}{|c|}{ Pseudomonas } \\
\hline SUMINISTRO & 0 & 0 & 0 & 100 \\
\hline SUBTERRÁNEO & 18 & 0 & 4 & 90 \\
\hline
\end{tabular}

Fuente. Elaboración propia.

Se observa que en el tanque de suministro solamente se aislaron Pseudomonas, mientras que en el tanque subterráneo se aislaron además de las Pseudomonas, coliformes totales y Enterococcus.

En cuanto a los microorganismos presentes en las unidades odontológicas se encontró la identificaron los siguientes: Stenotrophomonas maltophilia, Serratia, Shigella sp, Klebsiella oxytoca, Acinetobacter iwofii, Staphylococcus saprophyticus, Staphylococcus aureus, Staphylococcus haemolyticus, Enterococcus faecium, Staphylococcus auricularis, Streptococcus pyogenes, Pseudomonas putida, Pseudomonas aeruginosa, Pseudomonas fluorescens, Enterobacter gergoviae y Klebsiella pneumoniae. En los tanques se identificó Enterococcus faecium, Streptococcus pyogenes, Pseudomonas aeruginosa, Acinetobacter iwofii y Stenotrophomonas maltophilia.

\section{Discusión}

Al comparar los resultados obtenidos en el presente estudio, con la Resolución 2115 del 2007 del Ministerio de la Protección Social (6), se encontró que la calidad del agua de estas unidades no cumple con establecido en la norma para coliformes totales, por lo tanto, deben emplearse medidas correctivas y preventivas para el mejoramiento de la calidad del agua.

Los resultados de este estudio, al igual que en los resultados presentados por Muñoz en 2002, evidencian un recuento de UFC/ $100 \mathrm{~mL}$ de coliformes totales en la pieza de mano y en la jeringa triple (3). Las piezas de mano se contaminan internamente y el material depositado en el interior puede ser eliminado durante la operación, la pieza de mano y 
la jeringa triple no se esterilizan entre paciente y paciente, lo que facilita la transmisión de microorganismos tanto de los instrumentos como del agua que circula por estos (7).

El microorganismo que con mayor frecuencia se identificó fue la Pseudomonas aeruginosa, que se comporta como patógeno oportunista (8). Según la OMS, los microorganismos de la familia Pseudomonadaceae tienen la capacidad de proliferar en los conductos de agua de las unidades odontológicas y permanecer en ellos por largo tiempo (8). Otro aspecto importante es que estos microorganismos tienen la capacidad de inhibir los coliformes, por lo que se corre un gran riesgo de contaminación por agua con ausencia de coliformes, pero que en realidad podrían estar inhibidos por Pseudomonas (9-13).

El hecho de que Pseudomonas sea el microorganismo más aislado se puede explicar por la capacidad que tiene de formar biopelículas (14), debido a la producción de polisacáridos como el alginato y polímeros de glucosa, los cuales le confieren una gran capacidad de adherencia para la formación de bio. (15).

En el estudio de Falkinham y colaboradores, al igual que en el presente estudio, se reporta la presencia de Pseudomonas aeruginosa y microorganismos pertenecientes al grupo coliformes en tanques de almacenamiento de agua (16).

En tres de las cuatro muestras provenientes de las salas de cirugía, se obtuvo recuentos de 300 UF$\mathrm{C} / 100 \mathrm{~mL}$ para Pseudomonas, además en las piezas de mano de estas unidades se evidenció la presencia de Enterococcus, en estas salas se realizan procedimientos de carácter invasivo que podrían facilitar la entrada de los microorganismos y aumentar el riesgo de infección.

Shigella sp. fue uno de los microorganismos aislados. Es muy importante resaltar su presencia en el agua de las unidades odontológicas ya que es un indica- dor de contaminación fecal y no ha sido reportado en ningún estudio similar. La shigelosis se caracteriza por una diarrea acuosa, que puede progresar a deposiciones mucoides y sanguinolentas, causa una intensa reacción inflamatoria que se caracteriza por formación de abscesos y ulceración (17). Aunque se considera que la principal vía de infección es fecal-oral por contacto persona a persona, también se han reportado brotes de diarrea asociada a Shigella.

El presente estudio permitirá establecer las medidas preventivas y correctivas tanto en el manejo del agua como para el uso de los instrumentos de trabajo como la jeringa triple y la pieza de mano, con el fin de disminuir los riesgos de infección tanto para el personal de salud como para los pacientes y usuarios de esta (18-21).

\section{Conflicto de intereses}

Los autores declaran que no tienen conflicto de interés.

\section{Referencias}

1. Ávila S. Estupiñán M. Estupiñán D. 2012. Calidad del agua de unidades odontológicas. Nova 10; (17): 101-110

2. Shailee F, Sunite G. 2015. Microbial contamination of dental unit water line. in H.P. Government Dental College, Shimla. The Saudi Journal for Dental Research 6, 129-13

3. Walker J, Bradshaw D, Bennett A. 2000. Biofilm Formation and Contamination of Dental-Unit Water Systems in General Dental Practice Applied and Environmental Microbiology. 66, 3363-3367.

4. Chate R. 2006. An audit. Improves the quality of water within the dental unit water lines of the three separate facilities of a United Kingdom NHS trust. British dental journal. 201 (9): 565-569

5. Method 1604: Total Coliforms and Escherichia coli in Water by Membrane Filtration Using a Simultaneous Detection Technique (MI Medium) [en línea] 2002. Disponible en: http:// www.epa.gov/nerlcwww/1604sp02.pdf

6. Resolución 2115 de 2007. [en línea] Disponible en: http:// www.cra.gov.co

7. Monteiro M. 2003. Microbial Contamination in Dental Unit Waterlines. Braz Dent J. 14 (1): 55-57

8. Luján A. 2014. Pseudomonas aeruginosa: un adversario peligroso. Acta Bioquím Clín Latinoam 2014; 48 (4): 465-74 
9. Márquez Gómez Marco Antonio, Gómez Díaz Graciela María. Accidente ofídico en el departamento de Sucre, Colombia. Nova. 2015; 13 ( 24 ): 39-46.

10. Corrales Lucia Constanza, Antolinez Romero Diana Marcela, Bohórquez Macías Johanna Azucena, Corredor Vargas Aura Marcela. Bacterias anaerobias: procesos que realizan y contribuyen a la sostenibilidad de la vida en el planeta. Nova. 2015; 13( 24 ): 55-81.

11. González Yuri Lilian. Evaluación de la percepción del riesgo ocupacional en trabajadores de una empresa del sector de la construcción en Bogotá D.C. Nova. 2015; 13( 23 ): 93-107.

12. Carrero Sandra Helena Suescún, HerediaMontoya Dina Paola, Bolaños Yoryany Mulato, Medellín Martín Orlando Pulido. Seroprevalencia de infección por Leptospira y factores de riesgo en estudiantes de una universidad de Colombia. Nova. 2017; 15 ( 27 ): 131-138.

13. Naranjo Flórez Ricardo Andrés. Avances y perspectivas en Síndrome de Asperger. Nova. 2014; 12( 21 ): 81-101.

14. Rivera JA, Roman MC. Biopelículas y salud pública. 2005. Anales médicos. 50 (4): 172-176

15. Rasamiravaka T, Labtani Q, Duez P and El Jaziri M. 12015. The Formation of Biofilms by Pseudomonas aeruginosa: A Review of the Natural and Synthetic Compounds Interfering with Control Mechanisms. BioMed Research International. $17 \mathrm{p}$

16. Falkinham J, Pruden A and Edwards M. 2015. Opportunistic Premise Plumbing Pathogens: Increasingly Important Pathogens in Drinking Water. Pathogens. 4, 373-386

17. Chouhan S. 2015. Recovery of Salmonella and Shigella isolates from drinking water. European Journal of Experimental Biology. 5(7):49-61

18. Zuluaga Martha, Robledo Sebastian, Osorio-Zuluaga German A, Yathe Laura, Gonzalez Diana, Taborda Gonzalo. Metabolomics and pesticides: systematic literature review using graph theory for analysis of references. Nova. 2016; 14( 25 ): 121138.

19. Ávila de Navia Sara Lilia, Estupiñán-Torres Sandra Mónica, Díaz González Liliana. Calidad bacteriológica del agua Vereda El Charco, San Miguel de Sema, Boyacá- Colombia. Nova. 2016; $14(25)$ : 139-145.

20. Almonacid Urrego Carmen Cecilia, Camarillo Romero María del Socorro, Gil Murcia Zulay, Medina Medina Claudia Yasmin, Rebellón Marulanda Jennifer Viviana, Mendieta Zerón Hugo. Evaluación de factores de riesgo asociados a enfermedad cardiovascular en jóvenes universitarios de la Localidad Santafé en Bogotá, Colombia. Nova. 2016; 14(25): 9-17.

21. González Devia Johanna L., Monroy Romero Paola A., Almonacid Urrego Carmen C.. Homocisteína y otros factores de riesgo cardiovascular en niños de educación básica primaria del Colegio Distrital Manuel Elkin Patarroyo, Bogotá, D.C. Colombia. Estudio piloto. Nova. 2017 ; 15 ( 27 ): 103-117. 